\title{
An Agent Behavior Technique in an Autonomous Decentralized Manufacturing System*
}

\author{
Yasuhiro SUDO**, Nobuyuki SAKAO** and Michiko MATSUDA** \\ ** Kanagawa Institute of Technology \\ 1030 Shimo-Ogino, Atsugi, Kanagawa, 243-0292, Japan \\ E-mail: sudo@ic.kanagawa-it.ac.jp
}

\begin{abstract}
In this paper, we propose a technique for the communication between agents in the dynamic generation of a production plan in an autonomous production system using product agents, parts agents and assembly machine agents. A parts agent leads the promotion of processes in this type of assembly system. First, product agents create the assembly process model that contains the assembly sequence to complete the product. Next, product agents generate parts agents that have the assembly process model. Parts agents will be assembled by machines, with some communications between other agents. The proposed system is defined as an event driven architecture. The increase in the costs of communication between agents is polynomial in the number of the agents. Moreover, this system adjusts the production schedule dynamically using only local negotiation when conditions are changed. Finally, we show experimental results with simply examples.
\end{abstract}

Key words : Autonomous Decentralized Manufacturing System, Agent Based Production, Event Driven Architecture, Dynamic Scheduling

\section{Introduction}

A manufacturing system is evolving to attend the needs of the customer, mass production, a high-mix very-low-volume production and flexible order-made production. Therefore production planning is becoming increasingly more complex, there are, however, various study results concerning scheduling. These techniques usually consist of a mathematical formulation and an optimization of objective functions. ${ }^{(1)}$ Meanwhile, flexible autonomous manufacturing systems (Sugimura, et al., 2003) ${ }^{(2)}$ have no managing computer to generate commands, plans and schedules. In the system, production machines decide the work itself, with communicating with other machines. Moreover an autonomous manufacturing system with product parts agents has the potential for flexibility and robustness ${ }^{(3)(4)}$. That is to say that each agent behave reactively from various emergent events in real-time. However this manufacturing system has some problems in the communication and negotiation with other agents. In this paper, we discuss a technique for communication and negotiation between agents, in an autonomous decentralized manufacturing system with product parts agents and machine agents. The proposed system is defined as an event driven architecture. The increase in the costs of communication between agents is polynomial in the number of agents. Moreover, this system adjusts the production schedule dynamically using only local negotiation when conditions have to be changed. This paper is organized as follows. Section 2 contains review the autonomous decentralized manufacturing system with agents. In Section 3, a technique for a communication and negotiation between agents is described. In Section 4, there are some evaluations by the proposed technique, using examples. Section 5 contains a summary of our work and directions for future work.

*Received 31 Oct., 2009 (No. 09-0656) [DOI: 10.1299/jamdsm.4.673]

Copyright (c) 2010 by JSME 


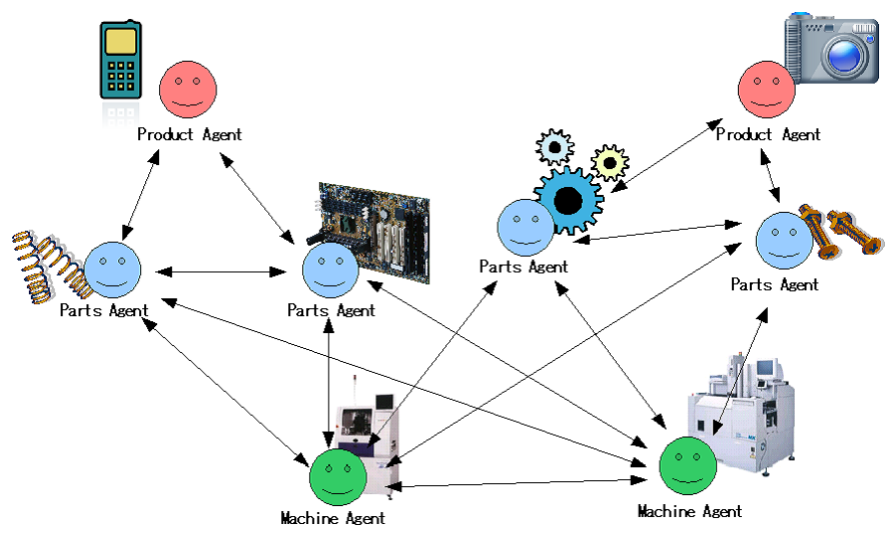

Fig. 1 The agent based autonomous decentralized assembly system

\section{Autonomous Decentralized Manufacturing System}

In the autonomous manufacturing system, a production plan is generated autonomously and dynamically, using communication and negotiation between agents that correspond to factory components. The structure of a traditional manufacturing system is a device oriented structure. There are several attempts to construct such kind of autonomous decentralized manufacturing system. One of example is the holonic manufacturing system ${ }^{(1)(5)(6)}$. The elements of the system act autonomously and organize the system consequently. And usually some manager functionality is installed as an agent or blackboard. If the manufacturing system doesn't need the manager function that manages the entire system by controlling each agent, it becomes a more flexible system. This means that the system should be constructed as an event driven type system. As a solution for the above mentioned requirements, configuration of a workpiece agent and a machine tool agent for an autonomous machining system has been introduced (Matsuda, et al., 2006) ${ }^{(3)}$. In the manufacturing systems, a manufacturing activity unit such as a machine tool, assembly machine, robot, AGV (Automatic Guided Vehicle), and manufacturing cell has autonomous functionalities that are configured as agents. In these cases, the system structure has been a device driven system structure.

On the other hand, the autonomous assembling system using parts agent has been introduced (Sakao, et al., 2008) ${ }^{(7)}$. In this system, the parts agent leads assembling process. And more, the product agent plans the processes and allocates the machine for executing the manufacturing processes required to turn the workpiece into the target product. These approaches different from this paper, are as follows. The parts are categorized into a main part that is based on assembly operation and sub parts that are assembled with the main part. However, it is surreal to provide part agents for every part that is just assembled with the other parts. For example, as of 2009, over 500 pieces of electronic parts on average are used for one cellular phone. So we do not use sub part agents in this paper, the part agents correspond only to the main parts. Instead, the product agents that create the assembly process model are allowed (Fig 1). The total size of main parts in each step is sufficiently-small compared to the size of assembled parts (Fig 2), thus reducing the costs of the communication between agents.

Considering that the implementation of these technique for real world factories, it is necessary to install the agent on each elements (assembly machines, products, component parts). The strategy to achieve this purpose is to use a RFID tag such a kind of wireless sensor module for example. Or more specifically, there are strategies that add the agent's capabilities to the carrying case (pallet) of products and component parts.

Meanwhile, if the virtual factory based on above concept is constructed on a computer, this virtual factory provides for users the assembly simulation environment that is for testing to adjust some parameters (ex. layout changed, machine broken). If this assembling system is in operation at same time with an assembly simulation system, that makes decisions on resource assignments and object layouts. 


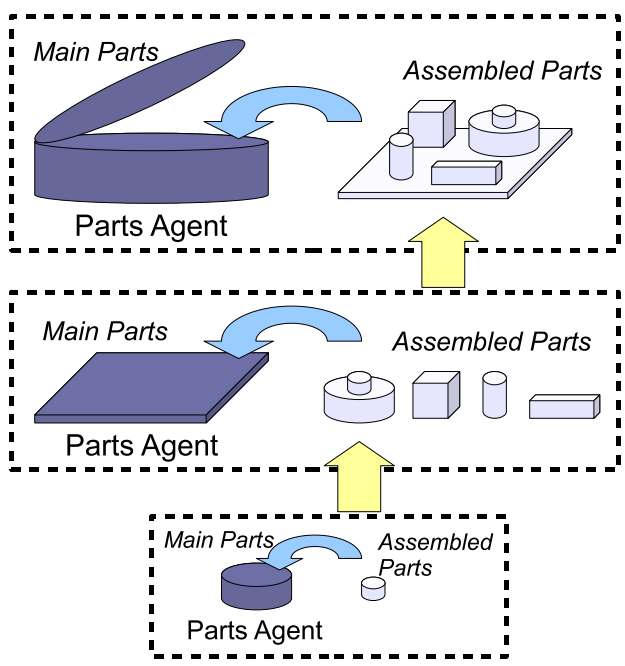

Fig. 2 The main parts and assembled parts

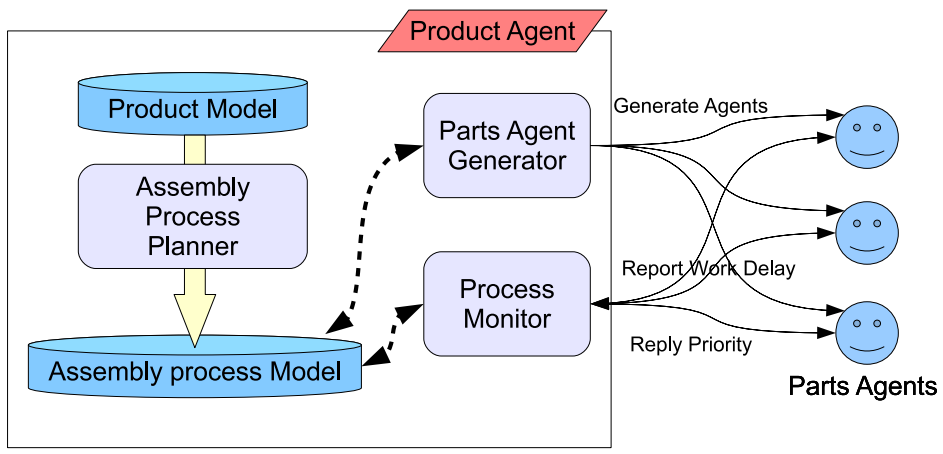

Fig. 3 The product agent

Agents used for the proposed assembly system are shown as follows.

\section{- The Product Agent}

When a new main part is input to the production line, the product agent makes an assembly process model from the product model which indicates the structure of the complete product (7). After this process, the product agent generates each parts agents that have process model, at the right time. While the product is unfinished, the product agent is monitoring parts agent's action, and communicating some information (work delay, changing deadline and etc.)(Fig 3).

\section{- The Parts Agent}

When the parts agent is dynamically generated by the product agent, a new main parts is input to the production line. The parts agents have assembly product plan which created by the product agent. In a process model, assembly operation plans, parts used, connection method, assembly angle, etc. are described, the agent then progresses its own assembly operation based on this process model. The agent disappears when a complete product or assembly parts have been achieved (Fig 4).

\section{- The Assembly Machine Agent}

The assembly machine agent has its own machine model that is described as specifications and capabilities of the assembly machine. The machine agent manages its own operation schedule using this machine model. Moreover, the machine agent has functions which checks and notifies the assembly machine's condition (Fig 5).

\section{Communication and Negotiations between Agents}

The autonomous decentralized manufacturing system has a large amount of agents, an efficient production plan is not generated if each agent decides greedily on its actions. In this section, we propose a method that improves the efficiency by suppressing the amount of the 


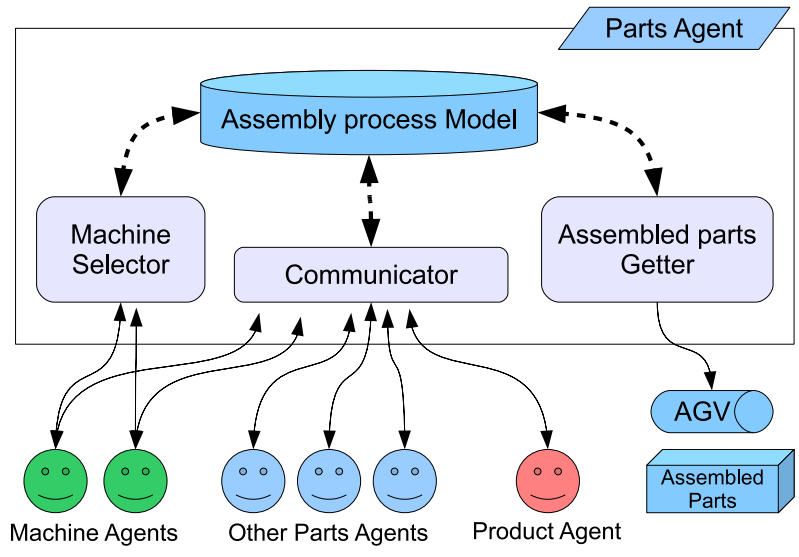

Fig. 4 The parts agent

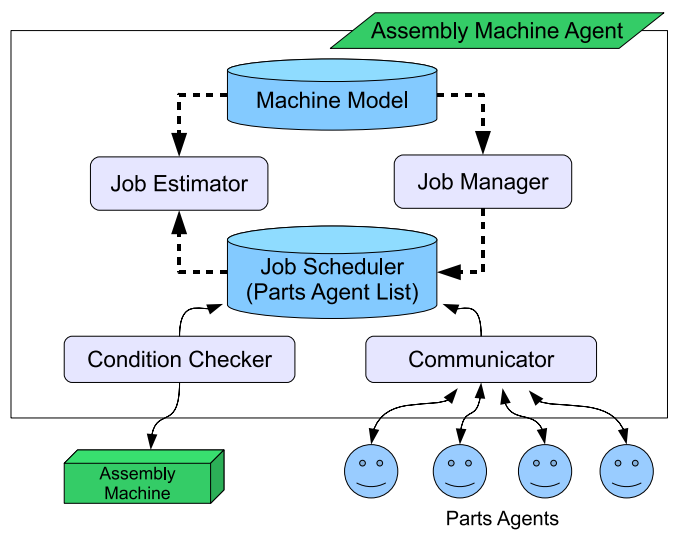

Fig. 5 The assembly machine agent

communication between agents.

\subsection{The Capabilities of communication for the Product Agent}

The product agent starts the event loop after creating the assembly process model. The required capabilities of communication for the product agent are defined as follows.

- Reply: When the inquiries from parts agent, the product agent reply the parts agent's priority.

- Catch: Catching the information from parts agents.

- Send: Sending a message that relating to the work delay and changing the assembly process model.

If each parts agent do works greedy, it is not efficient assembly production line. Each parts agent must know own priority for the assembly job. The priority is derived from Arrow Diagram (PERT Chart) obtained by the assembly process model. In case of comparison between two jobs in production lines for same product (Fig 6), it is enough to check only least node time (LNT) for deciding the job's priority. In Fig 6, job B has higher priority than job A. Meanwhile, in case of comparison between jobs for different products (Fig 7), it is necessary to consider not only LNT but also post assembly process and margin of deadline.

When some technical hitches are occurred, the parts agent transmits the information to the product agent. The product agent updates the assembly process model (PERT), and transmits the new process model to only relative agents. This means limiting influence on changing situation, to locally and minimum.

\subsection{The Capabilities of Negotiation for the Parts Agent}

The required capabilities of negotiation for the parts agent are defined as follows. 


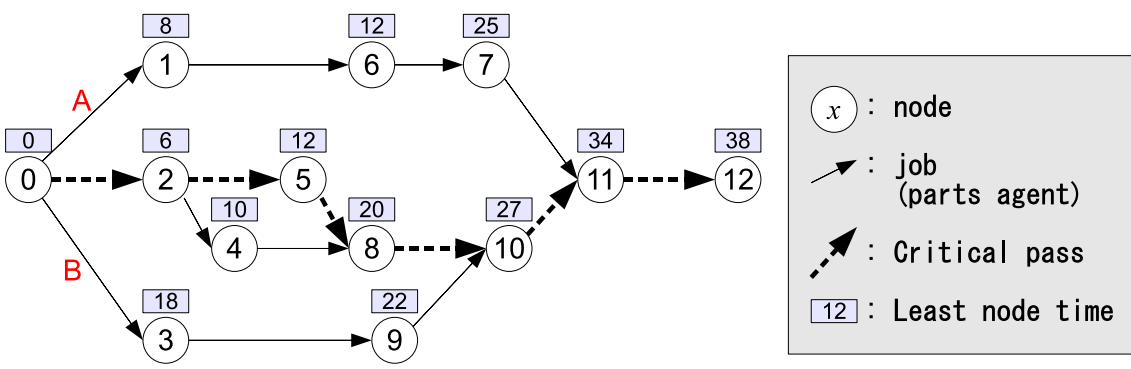

Fig. 6 Comparison of priority of job A and B (in the same product)

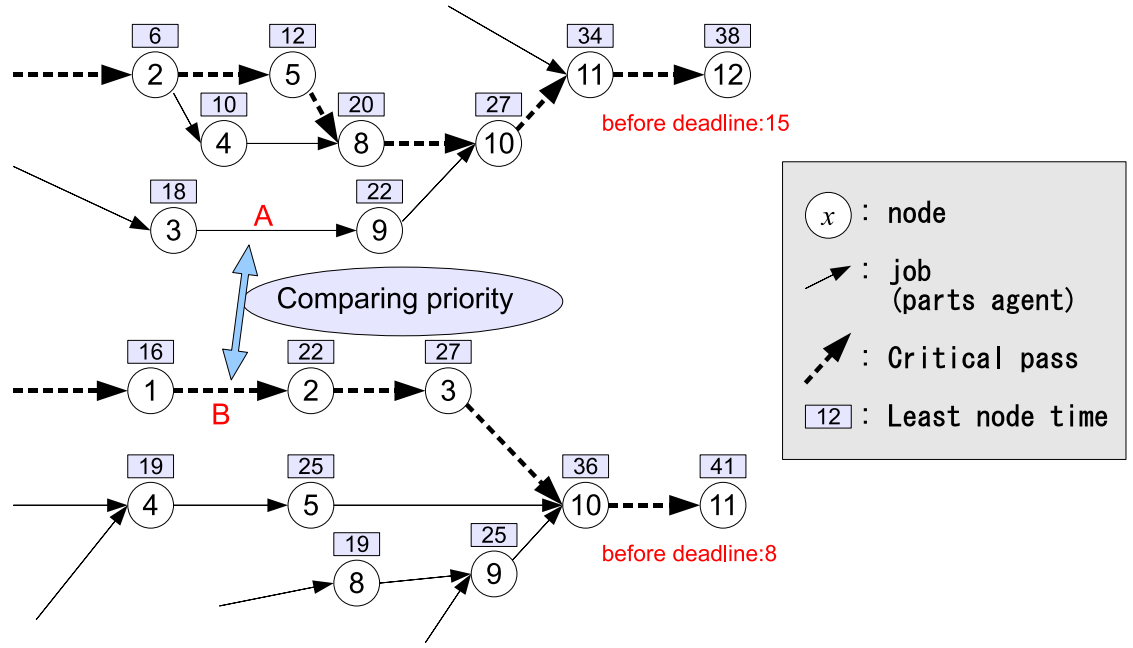

Fig. 7 Comparison of priority of job A and B (in different products)

- Ask: Sending inquiry for the possibility of assembly job to machine agents.

- Select: Formally sending assembly job request to the machine agent, after checking on the cost of transfer and finishing time.

- Change: Negotiation with other parts agents that are on the production line for the same machine schedule, for changing job order, and responding to it.

- Inspect: In Change process, it is used to know own priority.

- Switch: Sending a modified order which is generated by negotiations to machine agents.

- Get: Arranging the procurement of assembly parts, when the procurement authorization from the machine agent was received.

- Report: If it won't be able to finish the assembly job, the parts agent reports the delay to the product agent.

These capabilities that have an influence on generating a production plan are Ask, Select and Change. If the number of machine pieces is $|M|$, and the number of main parts pieces is $|P|$, then, if all of machines in the factory can handle all of the operations, the negotiation frequency is limited to $(|M|+1) \cdot|P|$ in the worst case. In the changing job order negotiation, the agent requests it for the other agent that is inferior in immediacy of the production's deadline or a time up to the following process, etc. This own priority is obtained by the product agent dynamically by using Inspect. However, it is not necessary in every Change. The priority is never refreshed until when the parts agent takes Send (the assembly process model is changed) from the product agent. After this time, the proposed method restricts the negotiation target to the adjacent agent on operation schedule list (queue). This means the agents perform a bubble sorting autonomously. So, the negotiation frequency is limited to $|L|^{2}$ for the length $\mathrm{L}$ of agent list on the machine agent. 


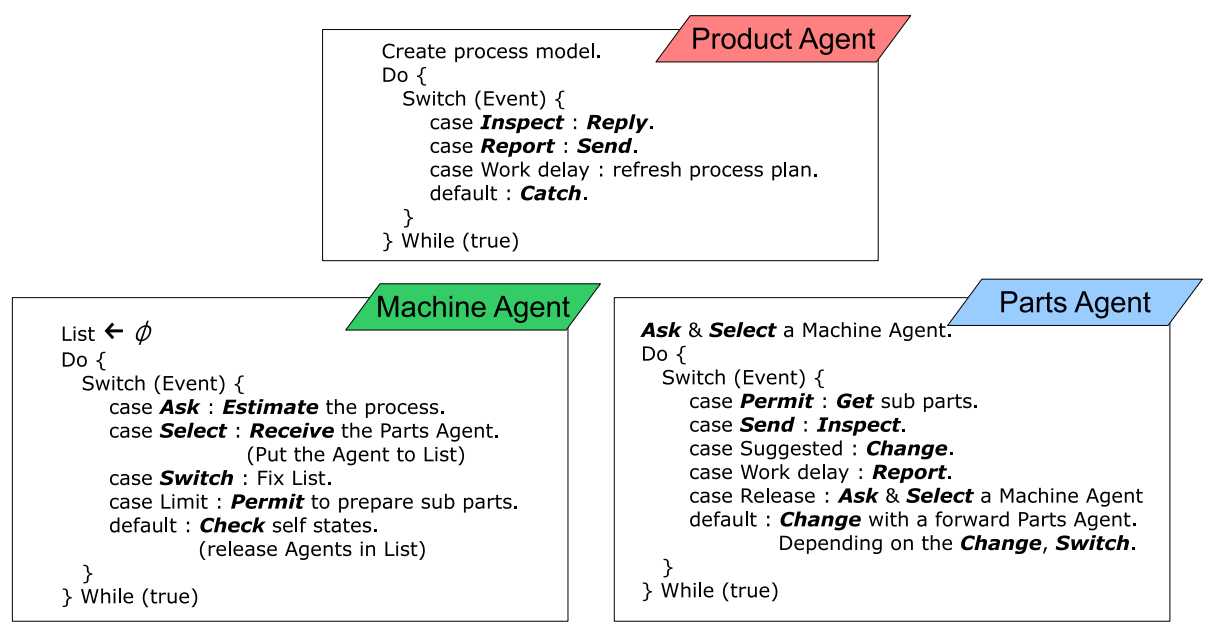

Fig. 8 The agent based production scheduling method

\subsection{The Capabilities of Negotiation for the Machine Agent}

The required capabilities of negotiation for the machine agent are defined as follows.

- Estimate: Sending estimated time of a beginning operation and a finishing operation by inquiry to its own schedule, when asked by the parts agents.

- Receive: Adding the job into its own operation schedule when selected by parts agents.

- Fix: Fixing the operation schedule when a request for switching job order from parts agents was detected.

- Permit: Permitting procurement of assembly parts for the parts agent which has imminent operation, and freezing the agent's schedule.

- Check: Checking the state of the assembly machine, if something unusual happens, apprising trouble status to parts agent. Releasing parts agents to own operation schedule, and rejecting subsequent requests.

The estimating capability refers to the assembly machine model. The assembly machine agent has an operation schedule such as a queue of parts agents, called List. The permitting procurement capability prevents changing the order and canceling at the last minute, and keeps the time of assembly parts procurement.

\subsection{The Autonomously Decentralized Dynamic Scheduling}

In this paper we propose an event-driven implementation as noted previously of the capabilities for each agent. Figure 8 shows the basic motion of assembly machine agents and parts agents. The assembly machine agent checks the state of the assembly machine and the list of parts agents while waiting for requests from parts agents. The parts agent repeats negotiation to other parts agents until the procurement authorization from the machine agent, after selecting an assembly machine.

Each agent decides the own action in response to events. When some technical problems or changing the due date are occurred, all of relative agents react to it dynamically. However in proposed system, if the new urgent work was put into assembling line, other agents never have to know it. The required communications and negotiations would be done locally.

\section{Example of System Working}

In this section, we verify the method by which the proposed system can achieve autonomously decentralized production scheduling by a one-to-one local communication, with a simple example.

Figure 9 shows the process that generated a parts agents' request for an operation with communication between machine agents (\#3). A parts agent starts to pick up an assembly machine agent (\#2) after generating the process model (\#1), meanwhile there is no communication between other parts agents. 


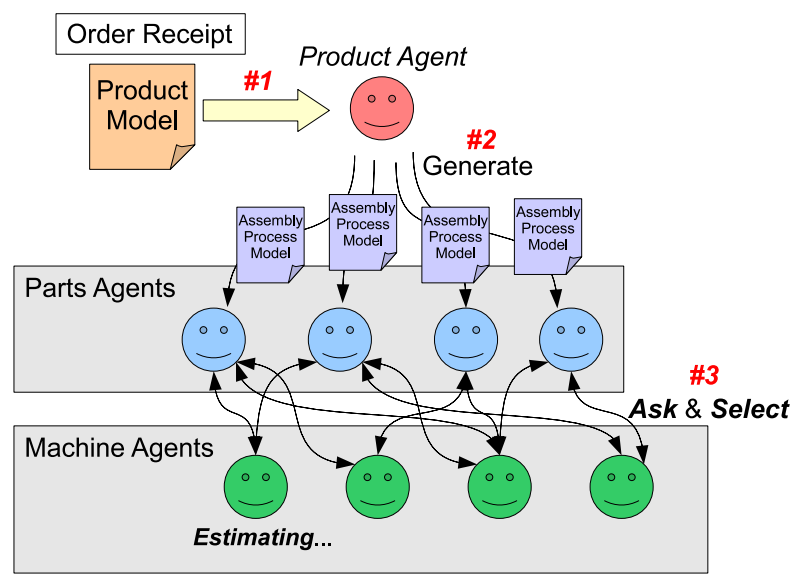

Fig. 9 Selecting an assembly machine

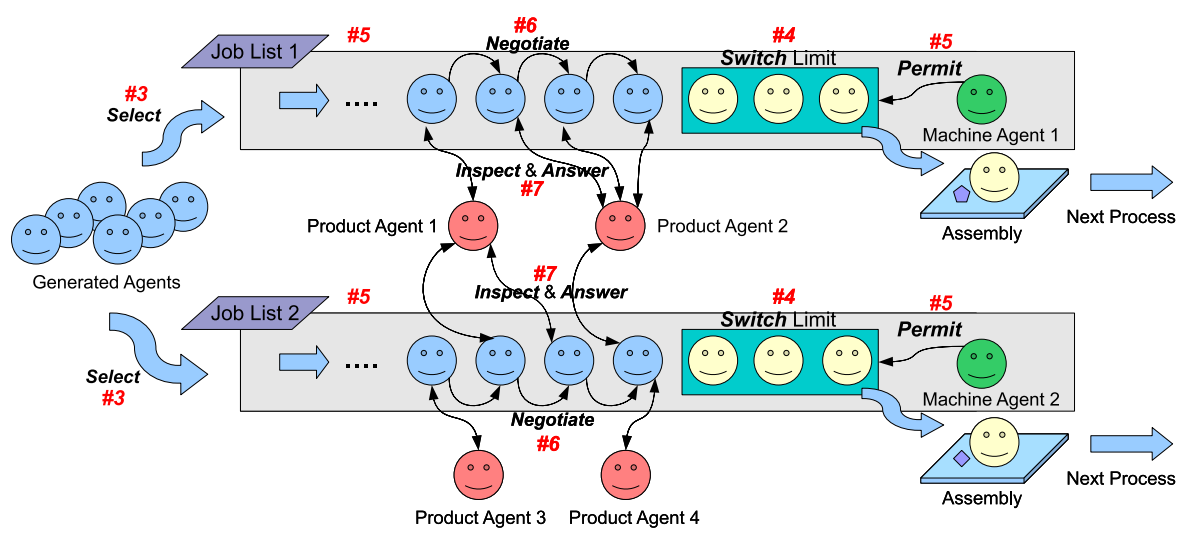

Fig. 10 The negotiation between parts agents

An assembly machine agent adds the job requested by the parts agent that is formally to be entered, into its own List (queue). When the parts agents in the List reach the limit for changing the schedule (\#4), the assembly machine agent permits procurement of assembly parts for the parts agent (\#5). The parts agent which obtained this permission procures the assembly parts necessary for assembly (\#10). The other parts agent such as one put lower in the List tries to change the job order by negotiation with parts agents higher in the List (\#6), until permission procurement authorization from the machine agent is obtained (Fig 10). In this process, the Inspect is not always called, it should be called only when the assembly process model is updated (\#7). Both parts agents make decision according to their assembly process model. If the Change was not invoked, the parts agent never tries Change between the same agents that have already negotiated.

The parts agent fixes the operation schedule List if a request for switching job order from parts agents was detected (\#8). However, the machine agent never involves itself in the Change between the parts agent and other parts agent (Fig 11). If the assembled parts agent is in mid-flow, it restarts at the beginning to select a new assembly machine. When the parts agent becomes a completed product or other assembled part, the agent begins the procedure for its disappearance (\#9).

If the state of an assembly machine would be unusable, or some unforeseen event occurred (\#11), the machine agent releases the parts agents on the operation schedule (\#12). The released parts agents then re-select another assembly machine (\#13) (Fig 12).

It was shown that an autonomous decentralized production plan was made possible in the above-mentioned example, by only using local negotiation between agents. 


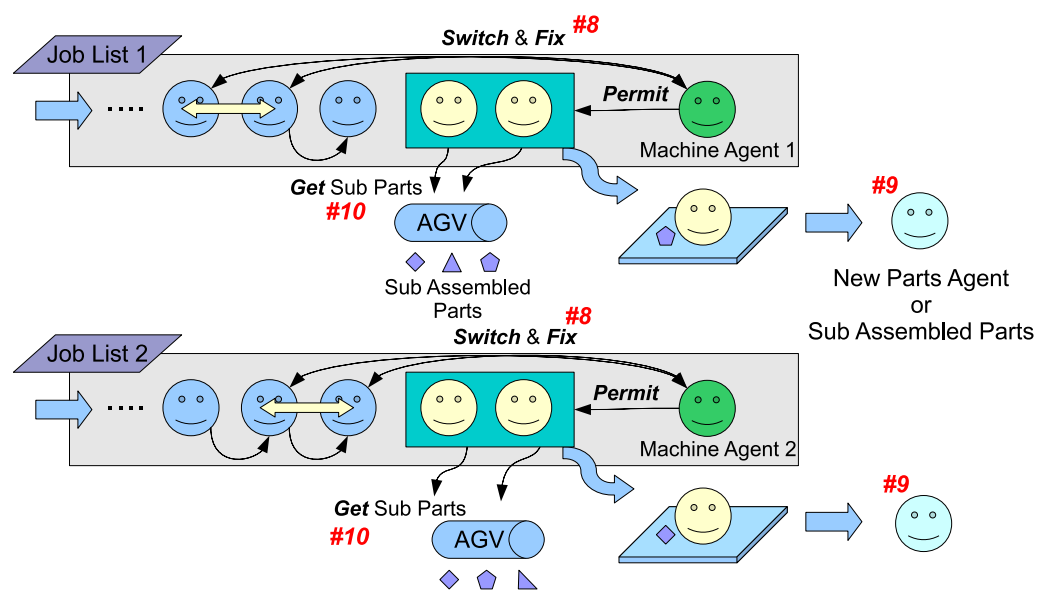

Fig. 11 Switching the parts agent's order

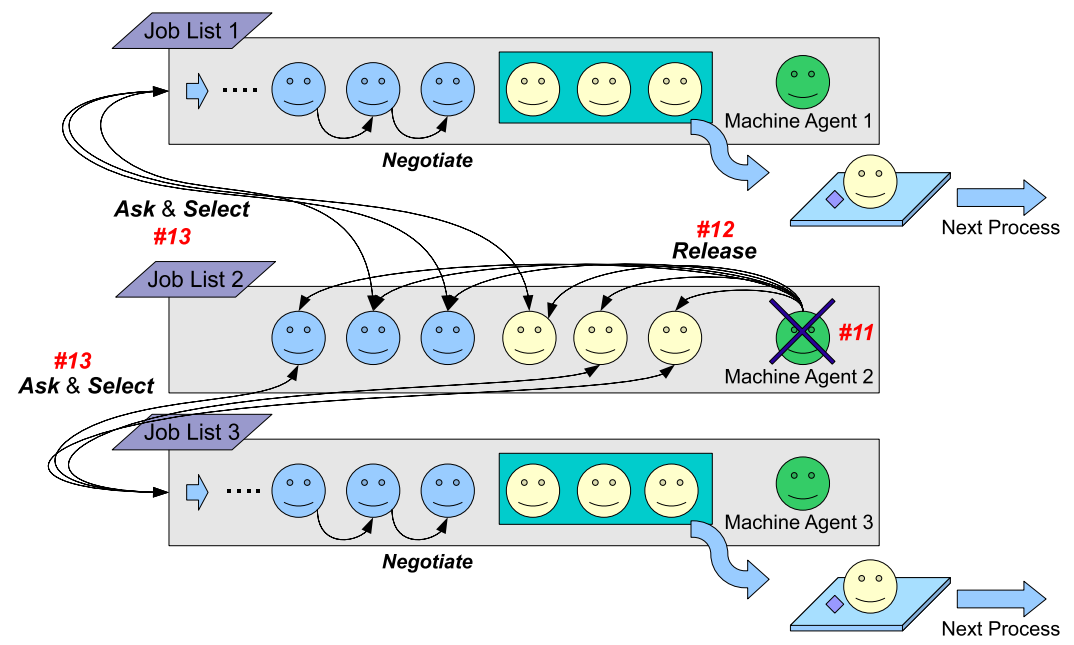

Fig. 12 Release and re-select a new machine

\subsection{The Dynamic Scheduling Simulation Results}

Figure 13 shows the charts that show example results of a job scheduling for regular production. Each job is described product type and lot's number. This example shows the changed schedule due to the input of a new parts. This chart starts at the time when main parts for a new lot of CP0 are put on the production line after 50th lot of FP has been put. Continuously, $\mathrm{CP} 1, \mathrm{CP} 2, \ldots$ are put on the production line. The conditions for job allocations and swapping are shown.

Figure 14 shows the changed schedule due to a machine breakdown. When Machine 1 is breakdown, the allocated jobs for the Machine 1 are released and they are taken back by the client part agent. These released jobs are reallocated to another machine which has the same ability as the broken machine. The re-allocation processes including swap are performed as in the regular allocation process by the parts agents.

\section{Conclusions}

We have proposed a method for the negotiation between agents in the dynamic generation of a production plan in an agent based autonomous decentralized production system. The method can be thought of as a new type of scheduling technique for autonomous decentralized production systems. If the above concepts are implemented as an autonomous assembling simulation system, it is a kind of virtual factory for use as real-time simulations and making decisions on resource assignments and object layouts. Future research topics include further improvement of efficiency (flexibility and robustness) and performance measurements using 


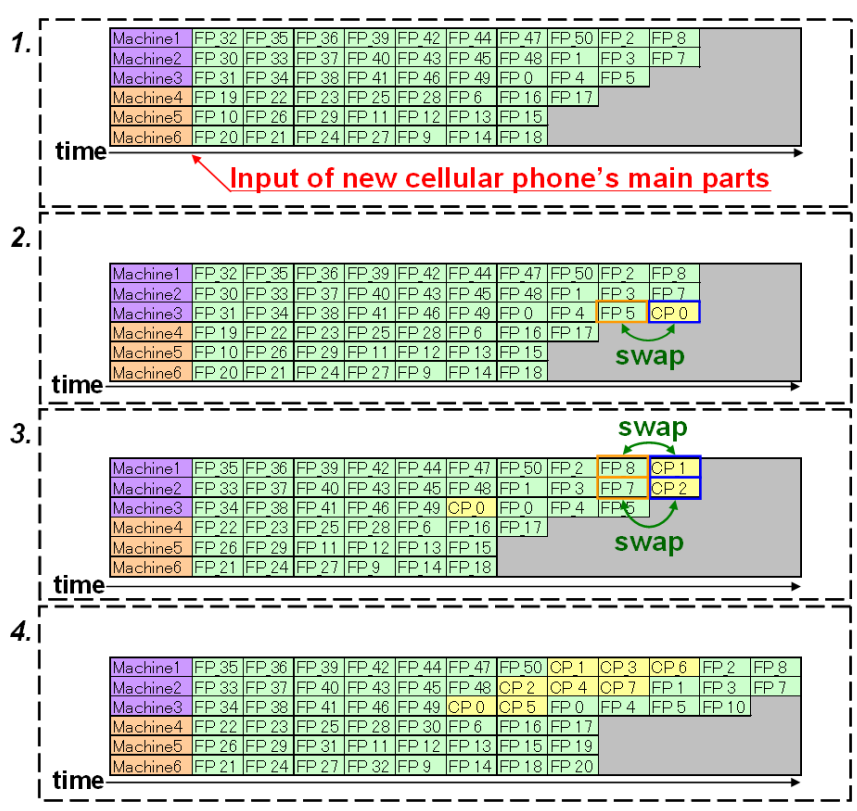

Fig. 13 Changed schedule due to input of new main parts

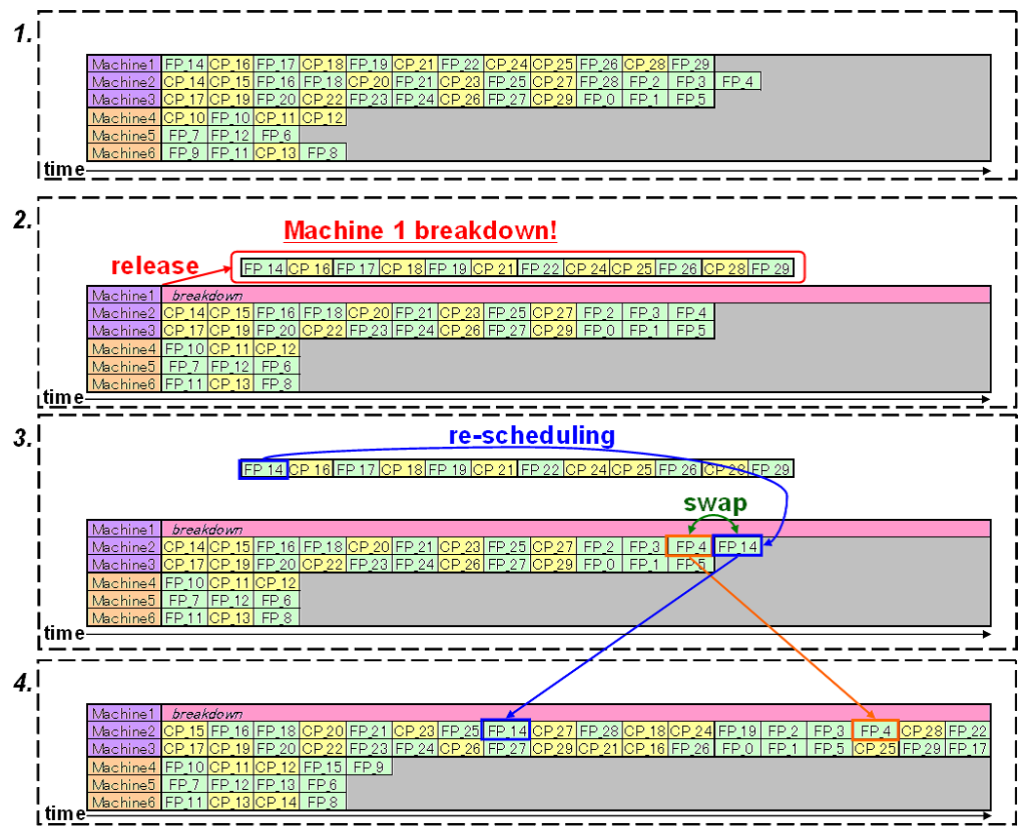

Fig. 14 Changed schedule due to machine breakdown

a real assembly simulation using wireless sensor network devices.

\section{Acknowledgements}

The authors are grateful to Dr. Udo Graefe, retired from the National Research Council of Canada for his helpful assistance with the writing of this paper in English.

\section{References}

( 1 ) Brussel, H. V., Wyns, J., Valckenaers, P., Bongaerts, L., and Peeters, P., "Reference architecture for holonic manufacturing systems: Prosa," Computers in Industry,, vol. 37 , no. 1, p. $255274,1998$.

( 2 ) Sugimura, N., Shrestha, R., and Inoue, J., "Integrated process planning and scheduling in holonic manufacturing systems -Optimization based on shop time and machining 
cost", Proc. of the 2003 IEEE International symposium on Assembly and task planning (ISATP2003), 2003, 36-41.

( 3 ) Matsuda, M., Ishikawa, Y., and Utsumi, S., "Configuration of Machine Tool Agents for Flexible Manufacturing", Proc. of The 39th CIRP-ISMS, 2006, 351-357.

( 4 ) Matsuda, M. and Sakao, N., "Configuration of An Autonomous Decentralized Digital Factory Using Product and Machine Agents", Innovation in Manufacturing Networks, IFIP vol.266, 2008, 215-222.

( 5 ) Fujii, N., Kobayashi, M., Makita, T., Hatono, I., Ueda, K., "Integration of Facility Planning and Layout Planning Using Self-Organization in Semiconductor Manufacturing", Proc. of the 37th CIRP-ISMS, 2004, 175-180.

( 6 ) McFarlane, D.C. and Bussman, S., "Developments in Holonic Production Planning and Control," Production Planning and Control, vol. 11, no. 6, 2000, pp. 522-536.

( 7 ) Sakao, N., Sudo, Y. and Matsuda, M., "Product and Machine Agents for an Autonomous Assembly Production System ", Proceedings of Joint 4th International Conference on Soft Computing and Intelligent Systems and 9th International Symposium on advanced Intelligent Systems, 2008, 1271-1276 